\title{
Manifestações bucais e considerações gerais da síndrome de Behçet: relato de caso
}

\section{Oral manifestations and general considerations of Behçet's Syndrome: case report}

\author{
Elen de Souza Tolentino* \\ Irma Milena Menck Romanichen** \\ Ariane Bessani ${ }^{* * *}$ \\ Laura Aline Nicácio ${ }^{* * * *}$ \\ Gustavo Zanna Ferreira****
}

\section{Resumo}

Objetivo: relatar um caso de síndrome de Behçet, enfatizando o controle das manifestações bucais. Relato de caso: paciente de 21 anos de idade, já diagnosticado com síndrome de Behçet há 7 anos, sem histórico familiar, relatatou o aparecimento de úlceras recorrentes, quando iniciou tratamento ortodôntico. Procurou a clínica odontológica da Universidade Estadual de Maringá queixando-se de "aftas e carne crescida" na boca. Foi realizada biópsia excisional do nódulo, e o exame histopatológico revelou tecido hiperplásico com infiltrado inflamatório crônico decorrente. Após 45 dias, observaram-se presença de novas aftas em mucosa labial, língua e gengiva assim como total cicatrização na região da biópsia. Atualmente, o paciente permanece em controle clínico médico e odontológico, estando as lesões aftosas presentes em diferentes regiões. Considerações finais: apesar de rara, essa doença é de grande relevância para o cirurgião-dentista, que deve saber diagnosticá-la e controlar suas manifestações bucais. Além disso, o acompanhamento é necessário para meIhorar a qualidade de vida do paciente.

Palavras-chave: Diagnóstico. Síndrome de Behçet. Tratamento.

\section{Introdução}

A síndrome de Behçet é uma doença inflamatória sistêmica caracterizada por várias manifestações clínicas, como: úlceras orais recorrentes, úlceras genitais, inflamações oculares, podendo envolver articulações, pele, sistema nervoso central, trato gastrointestinal, além de complicações pulmonares. ${ }^{1}$ É classificada como uma vasculite sistêmica, pois pode atingir artérias e veias de qualquer órgão. Sua etiologia permanece desconhecida, porém a hipótese mais aceitável da doença é a de que hospedeiros geneticamente susceptíveis desencadeiam uma resposta inflamatória causada por um agente infeccioso. ${ }^{2} \mathrm{O}$ fator de risco genético mais fortemente associado à síndrome de Behçet é o antígeno leucocitário humano (HLA)-B51, que ocorre em cerca de $60 \%$ dos pacientes com a doença. ${ }^{3}$

A síndrome de Behçet é mais frequente nos países ao longo da "Rota da Seda", uma rota de comércio antiga entre Ásia e Mediterrâneo Oriental, onde a prevalência do (HLA)-B51 é relativamente elevada em comparação com outras partes do mundo. ${ }^{4} \mathrm{~A}$ presença da doença na Turquia também é elevada. A doença é rara em indivíduos com idade superior a 50 anos e durante a infância. Apesar de ambos os gêneros serem igualmente afetados, nota-se que a doença é mais grave entre os homens jovens..$^{5,6}$

Não há nenhum teste específico para a doença de Behçet, e o diagnóstico é baseado em critérios clínicos. A história clínica detalhada é essencial para excluir outras condições com características pareci-

\footnotetext{
Doutora, professora das disciplinas Estomatologia e Radiologia, Universidade Estadual de Maringá, Maringá, PR, Brasil.

Aluna de graduação de Odontologia, Universidade Estadual de Maringá, Maringá, PR, Brasil.

Aluna de graduação de Odontologia, UniCesumar, Maringá, PR, Brasil.

Aluna de graduação de Odontologia, UniCesumar, Maringá, PR, Brasil.

Mestre, professor da disciplina Cirurgia e Traumatologia Bucomaxilofacial, UniCesumar, Maringá, PR, Brasil.
} 
das, como: síndrome de Reiter, sarcoidose e síndrome de Stevens-Johnson. ${ }^{2}$

$\mathrm{O}$ tratamento tem como objetivos o controle dos sintomas, a supressão precoce da inflamação e a prevenção de lesão de órgãos secundários. As opções de tratamento são agentes anti-inflamatórios e imunossupressores. As drogas são frequentemente utilizadas em combinação, a fim de maximizar a eficácia, minimizando os efeitos. $\mathrm{O}$ espectro de manifestações clínicas exige uma estreita cooperação multidisciplinar para o cuidado ideal. ${ }^{2}$

Frente ao exposto, o objetivo do presente estudo é relatar um caso de síndrome de Behçet, enfatizando o controle das manifestações bucais.

\section{Relato do caso clínico}

Paciente de 21 anos de idade, gênero masculino, leucoderma, já diagnosticado com síndrome de Behçet há 7 anos, sem histórico familiar, relatou o aparecimento de úlceras recorrentes, quando iniciou tratamento ortodôntico. Procurou a clínica odontológica da Universidade Estadual de Maringá queixando-se de "aftas e carne crescida" na boca.

$\mathrm{O}$ paciente relatou estar em acompanhamento com médico reumatologista desde o diagnóstico e fazer uso dos imunossupressores Adalimumabe (Humira ${ }^{\circledR}$ ) e Azatioprina, além de suplementos de vitamina $\mathrm{C}$ e $\mathrm{D}$. Adalimumabe era utilizado a cada 15 dias há 3 anos. Fez uso anterior de Colchicina, que foi suspenso pelo médico. $\mathrm{O}$ paciente não fazia uso de nenhum medicamento tópico para as lesões orais. As consultas de controle médico eram realizadas a cada 3 meses por conta dos efeitos adversos dos imunossupressores. $\mathrm{O}$ paciente relatou edema articular no joelho e problemas oftalmológicos graves (uveíte) antes de ter o diagnóstico estabelecido. Não relatava presença de ulcerações genitais ou cutâneas.

O exame físico extraoral não demonstrou alterações. Ao exame intraoral, foram observadas úlceras múltiplas na mucosa labial inferior e em língua, gengivite descamativa, além de um nódulo pediculado e ulcerado de aproximadamente $1 \mathrm{~cm}$ na região anteroinferior, indolor, com tempo de evolução aproximado de 3 meses (Figura 1). O paciente relatou uma afta previamente à lesão. Como as úlceras eram recorrentes e frequentes e o paciente não tinha queixas álgicas, sua principal preocupação era quanto ao crescimento em fundo de sulco.

Figura 1 - (A) Múltiplas úlceras em mucosa labial inferior; (B) Úlcera em língua; (C) Nódulo indolor pediculado e ulcerado em fundo de sulco vestibular na região anteroinferior; (D) Eritema em gengiva inserida na região anteroinferior (gengivite descamativa).
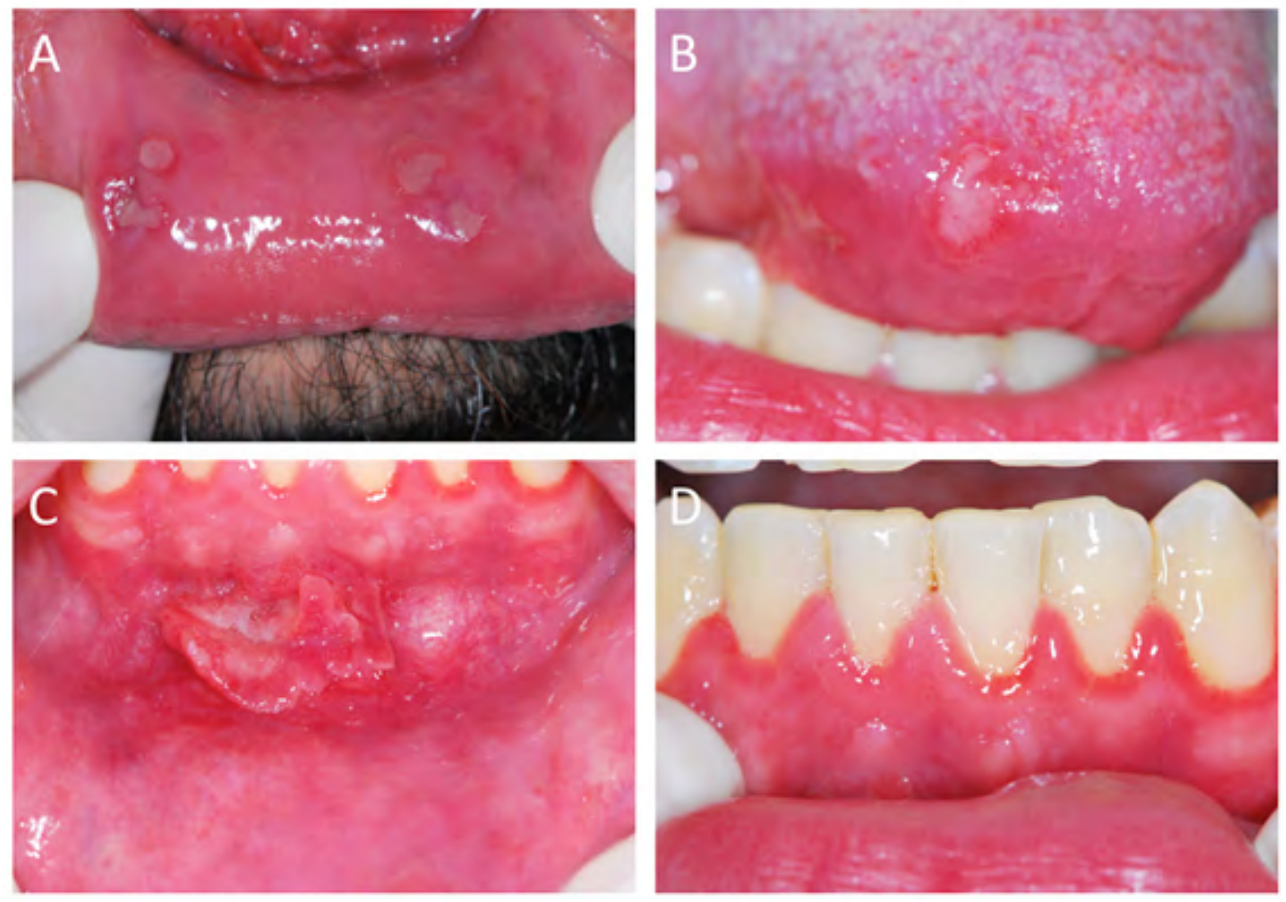

Fonte: Universidade Estadual de Maringá

Frente à história médica e ao diagnóstico prévio de síndrome de Behçet, estabelecido pelo reumatologista, as ulcerações orais foram diagnosticadas como aftas. Nesse momento, foi prescrito corticoide tópico (triancinolona acetonida em orabase) por 15 dias e acompanhamento. 
Após esse período, novas aftas em mucosa labial superior e inferior foram observadas, e não houve alterações no nódulo em fundo de sulco (Figura 2). O uso do corticoide tópico foi suspenso. Na ocasião, foi realizada biópsia excisional do nódulo (Figura 3), e o exame histopatológico revelou tecido hiperplásico com infiltrado inflamatório crônico, decorrente, possivelmente, de sucessivas remissões e exacerbações de úlceras na região (processo cicatricial).

Figura 2 - Controle de 15 dias: novas aftas em mucosa labial superior (A) e inferior (B) sem alterações na lesão nodular (B)
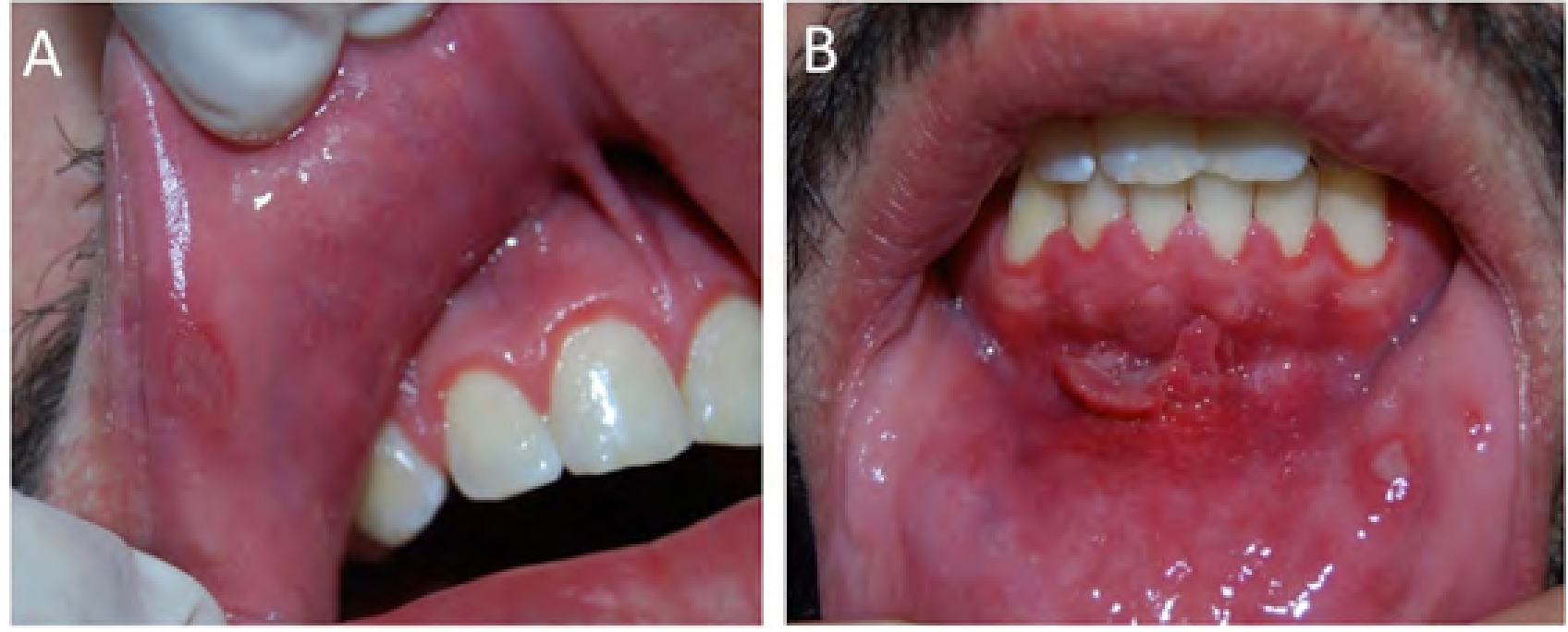

Fonte: Universidade Estadual de Maringá.

Figura 3 - Biópsia excisional do nódulo: (A) incisão; (B) remoção da lesão; (C) sutura; (D) peça encaminhada para exame histopatológico.
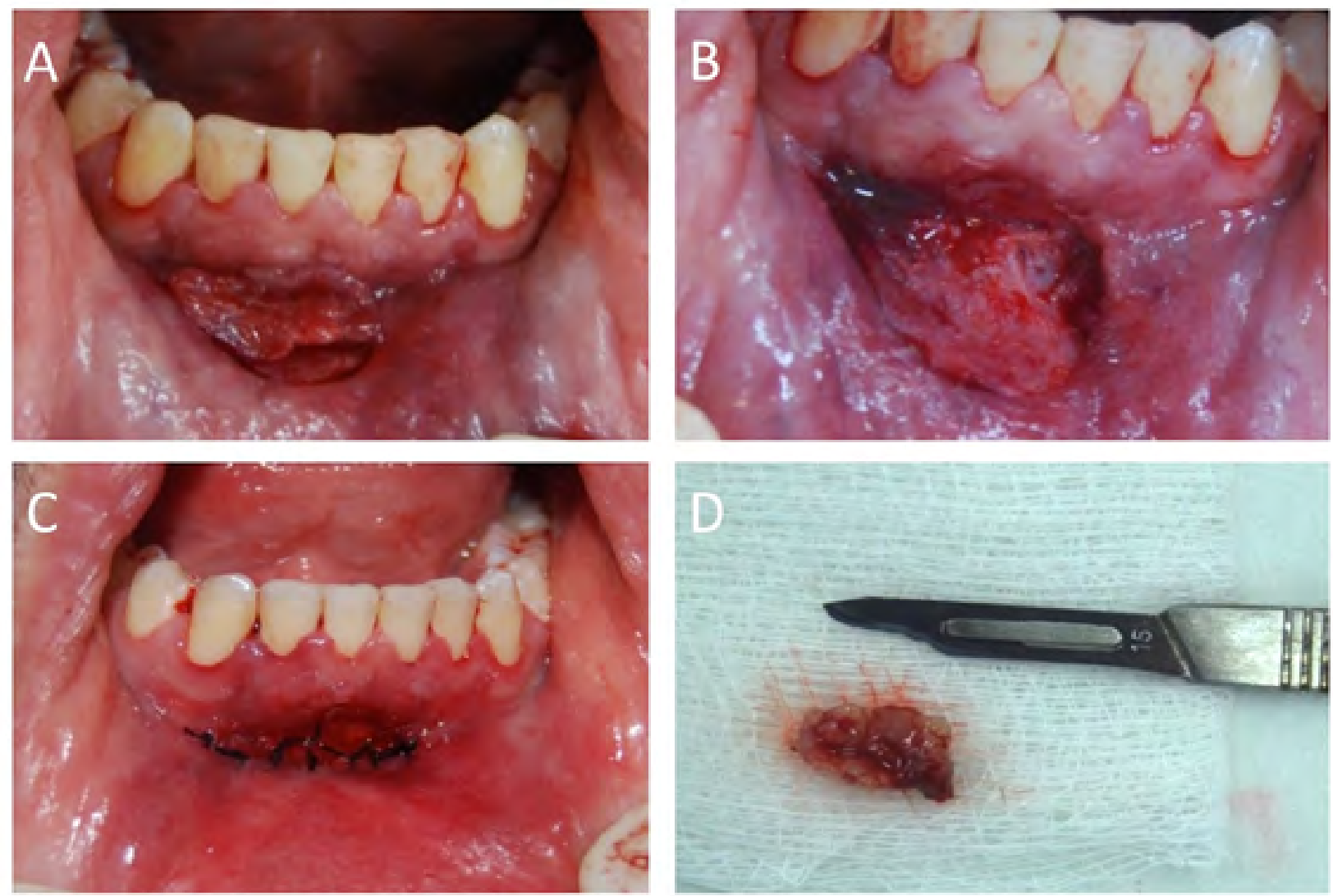

Fonte: Universidade Estadual de Maringá.

Após 45 dias, observaram-se presença de novas aftas em mucosa labial, língua e gengiva (Figura 4) e total cicatrização na região da biópsia. 
Figura 4 - Controle de 45 dias: presença de novas aftas em mucosa labial, gengiva (A) e língua (B)
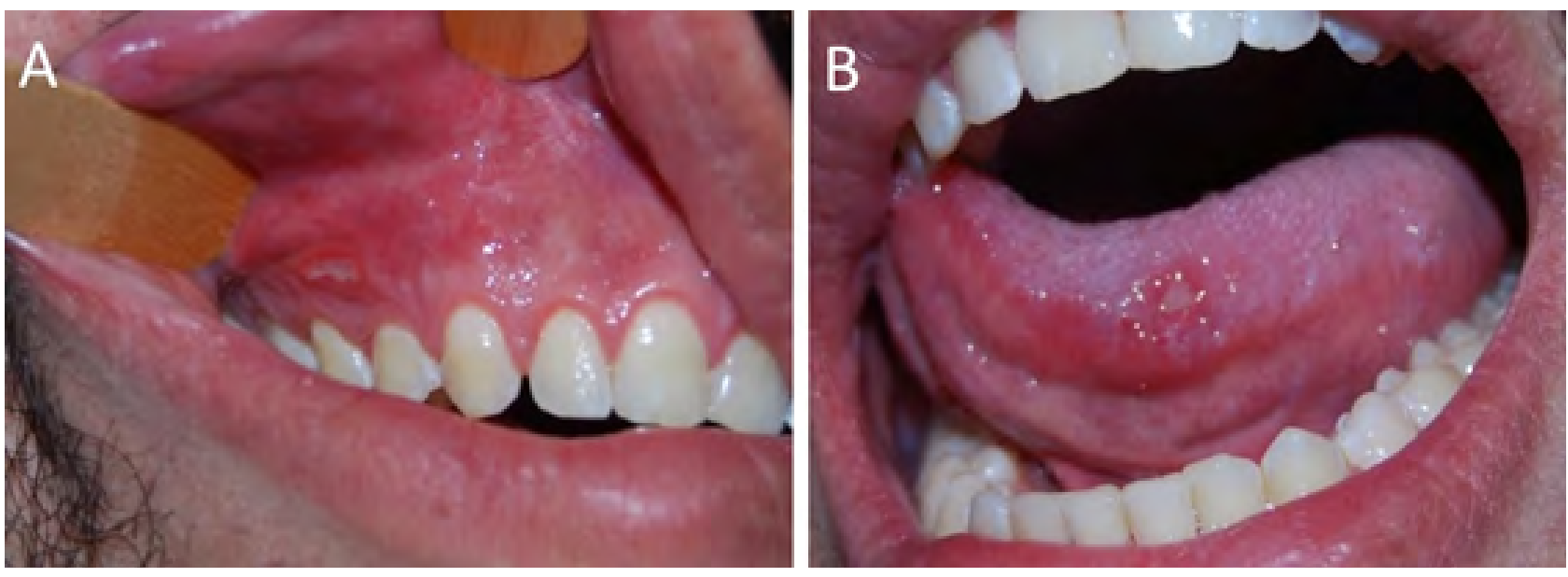

Fonte: Universidade Estadual de Maringá.

O paciente permanece em controle clínico médico e odontológico, estando as lesões aftosas presentes em diferentes regiões a cada consulta. Para que as imagens fossem publicadas, o paciente assinou $o$ termo de consentimento livre e esclarecido.

\section{Discussão}

A síndrome de Behçet, definida como uma doença multissistêmica de etiologia desconhecida e classificada como uma vasculite,${ }^{7}$ é caracterizada pela combinação de ulceração orais, genitais, lesões cutâneas e lesões inflamatórias dos olhos, sendo que as aftas correspondem a uma das características principais, podendo ser a manifestação mais precoce da doença. ${ }^{2} \mathrm{De}$ acordo com os critérios internacionais para a síndrome de Behçet ${ }^{8}$, um paciente é classificado como tendo a doença, quando acumula quatro ou mais pontos em um escore que varia de acordo com a manifestação (Quadro 1). A sensibilidade desse esquema foi relatada como $93,9 \%$, e a especificidade, como $92,1 \% .^{9}$

Apesar da etiologia desconhecida, acredita-se em uma forte ligação da doença com o antígeno (HLA)-B51, presente especialmente entre o Japão e o Mediterrâneo. ${ }^{7}$ A observação de casos familiares da doença sugere fortemente que os fatores genéticos desempenham um papel na patogênese, sendo a síndrome definida como uma doença poligênica. ${ }^{10}$ No presente caso, o paciente não relatou nenhum antecedente familiar.

A doença é mais comum entre a terceira e a quarta década de vida, mas também pode ser vista após os 50 anos de idade ou na infância..$^{5}$ Ambos os gêneros são igualmente afetados, mas a doença tende a ser mais severa em homens ${ }^{6}$ com idade inferior a 25 anos. ${ }^{5}$ Enquanto sinais oculares, lesões vasculares e lesões pustulosas são mais comuns nos homens, úlceras genitais e eritema nodoso são mais comuns nas mulheres. ${ }^{11,12}$ No caso relatado, o paciente recebeu o diagnóstico aos 14 anos de idade. $\mathrm{O}$ início dos sintomas ocorre com pouca frequência antes da puberdade ou depois dos 40 anos de idade. ${ }^{7}$

As úlceras orais e genitais observadas em na síndrome de Behçet são as mais comuns e, muitas vezes, os primeiros sinais da doença. ${ }^{13}$ No presente caso, embora as ulcerações orais fossem recorrentes, elas não foram as primeiras manifestações da doença, visto que o paciente foi diagnosticado pelo médico reumatologista, devido a fortes dores articulares.

O alívio das manifestações bucais pode ser feito com preparações tópicas de anestésicos locais (lidocaína $2 \%$ ) em aftas menores ou preparação local de esteroides que aderem à mucosa oral, como a pasta oral de triancinolona acetonida, que, aplicada localmente várias vezes ao dia, proporciona alívio dos sintomas e pode diminuir a duração da ulceração. ${ }^{7}$ Esta opção de tratamento tópico foi adotada no presente caso, durante 15 dias. A corticoterapia oral é utilizada na fase aguda da maioria dos pacientes e, nos casos mais leves, não é necessária. ${ }^{14}$ Apesar da diminuição exitosa da inflamação aguda, os corticoides sozinhos muitas vezes não conseguem evitar recaídas, sendo frequentemente usados em combinação com outros medicamentos, como no presente caso. $\mathrm{O}$ tratamento combinado também é usado para diminuir a dose de corticoides. ${ }^{15}$ Além disso, o uso a longo prazo desses medicamentos orais deve ser evitado, por causa dos efeitos adversos significativos. $^{2,16}$

Em caso de doença Behçet grave, os anticorpos monoclonais anti-TNF (fator de necrose tumoral) $\square$ Infliximabe e Adalimumabe $\square$ têm demonstrado grande eficácia. ${ }^{17}$ No caso, o paciente fazia uso de Adalimumabe, um anticorpo monoclonal unicamente humano, destinado a reduzir os sinais e sintomas, induzir resposta e remissão clínica, inibir a progressão dos danos estruturais e melhorar a capacidade física em pacientes adultos com artrite reumatoide ativa de intensidade moderada à grave. Além disso, 0 
paciente usava Azatioprina, um antimetabólito imunossupressor isolado. Seu uso é defendido somente se a ulceração oral for extremamente grave ou se existirem outras manifestações, como doença grave ocular, $^{7}$ como no caso do paciente, que desenvolveu uveíte prévia. Apenas Azatioprina ou a sua combinação com outras drogas imunossupressoras é um importante agente modificador da síndrome Behçet. ${ }^{2}$

Considerando que a ativação anormal de funções de neutrófilos foi reconhecida na patogênese da síndrome de Behçet, ${ }^{18}$ Colchicina tem sido amplamente utilizada como um fármaco básico para o tratamento da doença, visto que exerce efeitos benéficos na inibição das funções de neutrófilos, porém se mostra insuficiente para o tratamento de manifestações mais graves. Uma diminuição é observada na incidência de úlceras orais, genitais e lesões eritematosas no tratamento com Colchicina, assim como uma melhora nos sintomas da artrite. ${ }^{4,19}$

Em um estudo com 880 pacientes com síndrome de Behçet, descreveram-se as características oculares da doença. ${ }^{20} \mathrm{Em} 68 \%$ dos casos, os pacientes eram do gênero masculino, e a média de idade para o início da uveíte foi de 28,5 anos. Nas mulheres, o início foi aos 30 anos em média. O estudo também mostrou que lesões do polo posterior, mais alarmantes à visão, são mais comuns no gênero masculino. ${ }^{20}$ No presente caso clínico, o paciente já havia tratado uma uveíte grave, em que relatou quase ter perdido a visão, sendo esta sua manifestação clínica mais importante.

\section{Discussão}

A síndrome de Behçet, definida como uma doença multissistêmica de etiologia desconhecida e classificada como uma vasculite, ${ }^{7}$ é caracterizada pela combinação de ulceração orais, genitais, lesões cutâneas e lesões inflamatórias dos olhos, sendo que as aftas correspondem a uma das características principais, podendo ser a manifestação mais precoce da doença. ${ }^{2}$ De acordo com os critérios internacionais para a síndrome de Behçet $^{8}$, um paciente é classificado como tendo a doença, quando acumula quatro ou mais pontos em um escore que varia de acordo com a manifestação (Quadro 1). A sensibilidade desse esquema foi relatada como $93,9 \%$, e a especificidade, como $92,1 \% .^{9}$

Quadro 1 - Critérios internacionais para diagnóstico da síndrome de Behçet

\begin{tabular}{|c|c|}
\hline Sinal/Sintoma* & Pontos \\
\hline Lesões oculares & 2 \\
\hline úlceras genitais & 2 \\
\hline úlceras orais & 2 \\
\hline Lesões cutâneas & 1 \\
\hline Manifestações neurológicas & 1 \\
\hline Manifestações vasculares & 1 \\
\hline Teste de patergia positivo** & $1 *$ \\
\hline \multicolumn{2}{|c|}{$\begin{array}{l}\text { * Escore } \geq 4 \text { diagnóstico para a doença } \\
\text { ** O teste de patergia é opcional, e o sistema de pontuação primário não in- } \\
\text { clui este teste. No entanto, quando esse teste é conduzido, um ponto extra } \\
\text { pode ser atribuído para um resultado positivo. }\end{array}$} \\
\hline
\end{tabular}

Apesar da etiologia desconhecida, acredita-se em uma forte ligação da doença com o antígeno (HLA)-B51, presente especialmente entre o Japão e o Mediterrâneo. ${ }^{7}$ A observação de casos familiares da doença sugere fortemente que os fatores genéticos desempenham um papel na patogênese, sendo a síndrome definida como uma doença poligênica. ${ }^{10}$ No presente caso, o paciente não relatou nenhum antecedente familiar.

A doença é mais comum entre a terceira e a quarta década de vida, mas também pode ser vista após os 50 anos de idade ou na infância. ${ }^{5}$ Ambos os gêneros são igualmente afetados, mas a doença tende a ser mais severa em homens ${ }^{6}$ com idade inferior a 25 anos. ${ }^{5}$ Enquanto sinais oculares, lesões vasculares e lesões pustulosas são mais comuns nos homens, úlceras genitais e eritema nodoso são mais comuns nas mulheres. ${ }^{11,12}$ No caso relatado, o paciente recebeu o diagnóstico aos 14 anos de idade. $\mathrm{O}$ início dos sintomas ocorre com pouca frequência antes da puberdade ou depois dos 40 anos de idade. ${ }^{7}$

As úlceras orais e genitais observadas em na síndrome de Behçet são as mais comuns e, muitas vezes, os primeiros sinais da doença. ${ }^{13}$ No presente caso, embora as ulcerações orais fossem recorrentes, elas não foram as primeiras manifestações da doença, visto que o paciente foi diagnosticado pelo médico reumatologista, devido a fortes dores articulares.

O alívio das manifestações bucais pode ser feito com preparações tópicas de anestésicos locais (lidocaína $2 \%$ ) em aftas menores ou preparação local de esteroides que aderem à mucosa oral, como a pasta oral de triancinolona acetonida, que, aplicada localmente várias vezes ao dia, proporciona alívio dos sintomas e pode diminuir a duração da ulceração. ${ }^{7}$ Esta opção de tratamento tópico foi adotada no presente caso, durante 15 dias. A corticoterapia oral é utilizada na fase aguda da maioria dos pacientes e, nos casos mais leves, não é necessária. ${ }^{14}$ Apesar da diminuição exitosa da inflamação aguda, os corticoides sozinhos muitas vezes não conseguem evitar recaídas, sendo frequentemente usados em combinação com outros medicamentos, como no presente caso. $\mathrm{O}$ tratamento combinado também é usado para diminuir a dose de corticoides. ${ }^{15}$ Além disso, o uso a longo prazo desses medicamentos orais deve ser evitado, por causa dos efeitos adversos significativos. $^{2,16}$

Em caso de doença Behçet grave, os anticorpos monoclonais anti-TNF (fator de necrose tumoral) $\square$ Infliximabe e Adalimumabe $\square$ têm demonstrado grande eficácia. ${ }^{17}$ No caso, o paciente fazia uso de Adalimumabe, um anticorpo monoclonal unicamente humano, destinado a reduzir os sinais e sintomas, induzir resposta e remissão clínica, inibir a progressão dos danos estruturais e melhorar a capacidade física em pacientes adultos com artrite reumatoide ativa de intensidade moderada à grave. Além disso, o paciente usava Azatioprina, um antimetabólito 
imunossupressor isolado. Seu uso é defendido somente se a ulceração oral for extremamente grave ou se existirem outras manifestações, como doença grave ocular, ${ }^{7}$ como no caso do paciente, que desenvolveu uveíte prévia. Apenas Azatioprina ou a sua combinação com outras drogas imunossupressoras é um importante agente modificador da síndrome Behçet. $^{2}$

Considerando que a ativação anormal de funções de neutrófilos foi reconhecida na patogênese da síndrome de Behçet, ${ }^{18}$ Colchicina tem sido amplamente utilizada como um fármaco básico para o tratamento da doença, visto que exerce efeitos benéficos na inibição das funções de neutrófilos, porém se mostra insuficiente para o tratamento de manifestações mais graves. Uma diminuição é observada na incidência de úlceras orais, genitais e lesões eritematosas no tratamento com Colchicina, assim como uma melhora nos sintomas da artrite., ${ }^{4,19}$

Em um estudo com 880 pacientes com síndrome de Behçet, descreveram-se as características oculares da doença. ${ }^{20} \mathrm{Em} 68 \%$ dos casos, os pacientes eram do gênero masculino, e a média de idade para o início da uveíte foi de 28,5 anos. Nas mulheres, o início foi aos 30 anos em média. $O$ estudo também mostrou que lesões do polo posterior, mais alarmantes à visão, são mais comuns no gênero masculino. ${ }^{20}$ No presente caso clínico, o paciente já havia tratado uma uveíte grave, em que relatou quase ter perdido a visão, sendo esta sua manifestação clínica mais importante.

A escolha do tratamento da síndrome de Behçet depende da história da evolução da doença, das características clínicas das lesões e do seu grau de agressividade. O tratamento é necessário devido aos sintomas, e o papel do cirurgião-dentista é fundamental no reconhecimento das manifestações bucais, visto que elas podem ser o primeiro sinal da doença, que pode ter um curso grave, com diversas manifestações extrabucais. Úlceras orais recorrentes em qualquer fase da infância e da adolescência devem alertar para a possibilidade desse diagnóstico. $^{14}$

\section{Considerações finais}

A síndrome de Behçet é uma doença inflamatória sistêmica que muitas vezes tem como manifestação clínica inicial úlceras na cavidade oral, sendo de extrema importância que o cirurgião-dentista saiba diagnosticar e dar o correto encaminhamento. O tratamento com anticorpos monoclonais anti-TNF, Infliximabe, Adalimumabe e Colchicina, tem se mostrado eficaz. O acompanhamento se faz necessário para melhorar a qualidade de vida do paciente, visto que as lesões orais podem ser bastante dolorosas, e a combinação de medicamentos pode produzir efeitos adversos importantes.

\section{Abstract}

Objective: report a case of Behçet's Syndrome, emphasizing the control of oral manifestations. Case report: a 21-year-old patient, diagnosed with Behçet's Syndrome for 7 years, with no family history, reporting recurrent ulcers when orthodontic treatment was started. He sought a dental clinic at the State University of Maringá complaining of "aphthae and grown flesh" in the mouth. Excisional nodule biopsy and histopathological examination of hyperplastic tissue with chronic inflammatory infiltrate were performed. After 45 days, the presence of new aphthae were observed in the labial mucosa, tongue and gingiva, as well as total scarring in the region of the biopsy. Currently, the patient remains in clinical and dental control, remaining as aphthous interventions present in different regions. Final considerations: although rare, this disorder is of great relevance for the dental practioner, who must know how to diagnose and control its oral manifestations. In addition, follow-up is necessary to improve the patient's quality of life.

Keywords: Diagnosis. Behçet's syndrome. Treatment.

\section{Referências}

1. Tuzun H, Seyahi E, Guzelant G, Oz B, Batur S, Demirhan $\mathrm{O}$, et al. Surgical treatment of pulmonary complications in Behçet's Syndrome. In: Seminars in thoracic and cardiovascular surgery, 2018, [s. 1.]. Proceedings. 2018. p. 369-378.

2. Marshall S. Behcet's disease. Best Pract Res Clin Rheumatol 2004; 18(3):291-311.

3. Gul A. Standard and novel therapeutic approaches to Behcet's disease. Drugs 2007; 67(14):2013-22.

4. Yurdakul S, Mat C, Tüzün Y, Ozyazgan Y, Hamuryudan V, Uysal O, et al. A double-blind trial of colchicine in Behçet's syndrome. Arthritis Rheum 2001; 44(11):2686-92.

5. Yazici H, Tuzun Y, Pazarli H, Yurdakul S, Ozyazgan $\mathrm{Y}, \mathrm{Ozdo} \square$ an $\mathrm{H}$, et al. Influence of age of onset and patient's sex on the prevalence and severity of manifestations of Behçet's syndrome. Ann Rheum Dis 1984; 43(6):783-9.

6. Tursen U, Gurler A, Boyvat A. Evaluation of clinical findings according to sex in 2313 Turkish patients with Behçet's disease. Int J Dermatol 2003; 42(5):346-51.

7. Yazici H, Barnes CG. Practical treatment Recommendations for Pharmacotherapy of Behçet's Syndrome. Drugs 1991; 42(5):796-804.

8. International Team for the Revision of the International Criteria for Behçet's Disease (ICBD). The International Criteria for Behçet's Disease (ICBD): a collaborative study of 27 countries on the sensitivity and specificity of the new criteria. $\mathrm{J}$ Eur Acad Dermatol Venereol 2014; 28:338-347.

9. Davatchi F. Diagnosis/Classification Criteria for Behçet's Disease. Patholog Res Int 2012; 2012:607921.

10. Mendoza-Pinto C, García-Carrasco M, Jiménez-Hernández M, Jiménez Hernández C, Riebeling-Navarro C, Nava Zavala A, et al. Etiopathogenesis of Behçet's disease. Autoimmun Rev 2010; 9(4):241-5.

11. Davatchi F, Shahram N, Chams H. The influence of gender on the frequency of clinical symptoms in Behçet's disease. Adv Exp Med Biol 2003; 528:65-6. 
12. Bang D, Oh S, Lee KH, Lee ES, Lee S. Influence of sex on patients with Behçet's disease in Korea. Adv Exp Med Biol 2003; 528:59-63.

13. Ideguchi H, Suda A, Takeno M, Ueda A, Ohno S, Ishigatsubo Y. Behçet disease: evolution of clinical manifestations. Medicine (Baltimore) 2011; 90(2):125-32.

14. Albuquerque PR, Terreri MTRA, Lên CA, Hilário MOE. Doença de Behçet na infância. J de Pediatria 2002; 78(2):12832.

15. Mendes D, Correia M, Barbedo M, Vaio T, Mota M, Gonçalves $\mathrm{O}$, et al. Behçet's disease - a contemporary review. J Autoimmunity 2009 ; 32(3-4):178-88.

16. Mat C, Yurdakul S, Uysal S, Gogus F, Ozyazgan Y, Uysal O, et al. A double-blind trial of depot corticosteroids in Behcet's syndrome. Rheumatology 2006; 45(3):348-52.

17. Ferrão C, Almeida I, Marinho A, Vasconcelos C, Correia A. Nossa regra de ouro na doença Behçet: tratar a manifestação clínica. Arq Med 2015; 29(3):75-9.

18. Hirohata S, Kikuchi H. Behçet's disease. Arthritis Res Ther 2003; 5(3):139-46.

19. Davatchi F, Sadeghi Abdollahi B, Tehrani Banihashemi A, Shahram F, Nadji A, Shams H, et al. Colchicine versus placebo in Behçet's disease: randomized, double-blind, controlled crossover trial. Mod Rheumatol 2009; 19(5):542-9.

20. Tugal-Tutkun I, Onal S, Altan-Yaycioglu R, Huseyin Altunbas H, Urgancioglu M. Uveitis in Behçet's disease: an analysis of 880 patients. Am J Ophthalmol 2004; 138(3):373-80.

Endereço para correspondência:

Irma Milena Menck Romanichen Avenida Mandacaru, 1550, Centro 87080-000, Maringá, PR, Brasil

Telefone/Fax: (+55) (44) 3011-9052

E-mail: irmamilena34@gmail.com

Recebido: 21/08/18. Aceito: 27/09/18. 